\title{
Autonomous Braking System: for Automobile Use
}

\author{
Ataur Rahman ${ }^{*}$, Sany Izan Ihsan ${ }^{2}$ \\ Department of Mechanical Engineering, Kulliyyah of Engineering, International Islamic University Malaysia (IIUM), Kuala \\ Lumpur, Malaysia \\ Emails: *1arat@iium.edu.my; ${ }^{2}$ sihsan@iium.edu.my
}

\section{ARTICLE INFO}

\section{Article History:}

Received: $07^{\text {th }}$ October 2021

Revised: 23rd November 2021

Accepted: 24th November 2021

Published: 23 ${ }^{\text {rd }}$ December 2021

\section{Keywords:}

Autonomous braking

Advanced vehicle

Adaptive neuro-fuzzy interference

Genetic algorithm

Sensors bank

Uncertainty

\section{A B S T R A C T}

Road fatality and injury are a worldwide issue in the transportation industry. Road traffic accidents are becoming increasingly significant due to higher mortality, injury, and disability across the world, particularly in developing and transitional economies. Eighty-five percent of the total road traffic fatalities occur in developing nations, with Asia-Pacific accounting for roughly half of them. A variety of factors influence road safety, including technological, physical, social, and cultural factors. The purpose of this research was to design an autonomous braking system (AuBS). Using the Adaptive Neuro-Fuzzy Intelligent System (ANFIS), a DC motor, sensors, and SAuBS have been developed to customize the traditional hydraulic braking system. The genetic algorithm has been developed to simulate the fundamental characteristics of the automotive braking system. The AuBS system goal is to slow the car without the driver's help infrequent braking when the vehicle is moving at slower speeds. When the ANFIS performance is compared to that of the AuBS model, it is discovered that the ANFIS performs roughly $15 \%$ better.

(C) 2021 MIJST, All rights reserved.

\section{INTRODUCTION}

In the transportation sector, road fatality and injury are known global issues. Major research on the topic of road traffic accidents and fatalities has been emphasized by the World Bank, World Health Organization (WHO), Transport Research Laboratory (TRL), and others. Road traffic accidents are becoming increasingly important as a source of death, injury, and disability all across the world, particularly in developing and transitional economies (Mittal, 2008). Road traffic accidents entail not only enormous financial losses, but also severe physical and mental anguish for people, families, and businesses. According to WHO figures, road traffic accidents account for $75 \%$ of deaths in developing nations, despite the fact that they own only $32 \%$ of the world's vehicles. Road traffic accidents are expected to cost the world economy USD 518 billion each year.

Another major source of road traffic accidents and deaths in the country is the country's rapid expansion in the number of automobiles. Accidents happen because of the faults of drivers and other road users, or because of the faults of the vehicle. Human failure is caused by the driver's or the victim's guilt, alcoholic intoxication, exhaustion, irresponsible driving and not obeying the rules of the road, and ignorance of road safety, which result in an accident and death (Friduly, 1999).
The frightening of traffic accidents is a source of considerable concern for all of us. Eighty-five percent of road traffic deaths occur in developing nations, with AsiaPacific accounting for roughly half of them. According to Anbalagan and Suyambukanni (2010), India accounts for around $10 \%$ of all road traffic deaths worldwide. Accidents on the road are unforeseeable, but they are not unavoidable. Road safety is complicated by a number of aspects, including technological and physical, as well as social and cultural (Hassen et al., 2011).

In their study, the authors (Breuer et al., 2007) found that late and untimely driver intervention in braking, as well as insufficient braking torque obtained in emergency scenarios, are among the leading causes of road accidents.

Late braking was found to be caused by driver irresponsibility and human error by the European New Car Assessment Program (Euro NCAP) (Pain et al., 2011). Studies were conducted in the domain of active vehicle safety to lessen these issues, which led to the introduction of Advanced Driver Assistance Systems (ADAS) (Hamid et al., 2016). To address these difficulties, researchers in the field of vehicle active safety conducted research, which led to the creation of Advanced Driver Assistance Systems (ADAS). Collision avoidance (Hamid et al., 2017), blindspot monitoring, and automated emergency braking (AEB) (Fildes et al., 2015) are examples of notable ADAS 
applications. AEB is an ADAS feature that works autonomously, without the need for human interaction, to lessen the chance of a collision in dangerous situations by applying braking torque. Volvo City Safety (Distner et al., 2009), VOLKS WAGON Front Assist (Berger et al., 2015), and the 2010 Mercedes-Benz PRE-SAFER Brake (Zwahlen et al., 2016) are three AEBs that have been introduced on the road in the last five years.

Despite the vast number of commercial AEB products, crash events have occurred when the distance between the vehicle to vehicle is too near (Hong et al., 2013). To avoid this issue, an AEB system that considers and maintains a safe distance from the point of impact should be developed (Rahman et al., 2018). The ASEAN New Car Assessment Program (ASEAN NCAP) mentioned the AEB as a requirement in its new grading protocols for 2017-2020 (Asean nCAP, 2020).

Over time, innovators have added new features to the brake system with the goal of easing driving environments by improving braking performance. The evolution of brakes has been impressive, and it has incorporated many technological breakthroughs over the years (Rahman et al., 2018).

Nowadays, assist systems have diffused from high-end to low-cost automobiles, and they are known by various names such as advanced safety assist (ASA), smart sensing, advanced driver assist system (ADAS), and so on. As a result, automatic emergency braking (AEB) evolves, in which the car automatically applies brakes in a dangerous scenario without the assistance of the driver. The system integrates an ultrasonic sensor with a controller to identify if the vehicle is within a specified range. If the driver looks to be ignorant of the situation, the system will activate AEB to reduce the risk of accidents (Zwablen et al., 2016).

This research aims to present a fundamental autonomous braking system with the existing hydro-mechanical braking system, in which the brake pedal simply engages with an electromagnetic system based on the signals of the accelerator pedal sensor and the wheel speed sensor. The ANFIS controller has been proposed to control the power flow to the electromagnetic system since the fluctuating velocity necessitated varying pressure to maintain the vehicle's decelerating distance to a full stop.

\section{MATERIALS AND METHODS}

The development of the AuBS was carried out following the flow chart as shown in Figure 1. The dc servo-motor actuates the master cylinder to create the appropriate brake hydraulic pressure from the master cylinder (MC) for the creation of braking force at the wheel-calipers, as shown in the diagram. Hydraulic braking mechanism, dc servomotor, ANFIS controller, wheel speed and ultrasonic sensors, and a dc power source are the main components of the AuBS.

\subsection{Braking System Architecture}

Figure 2 depicts an AI-based IoT-controlled DC motorpowered autonomous electro-hydraulic braking system
(AEHBS). The DC motor supports the brake pedal force in this system, with the wheel speed sensor, brake controller, and proportional valve controlling the total system.

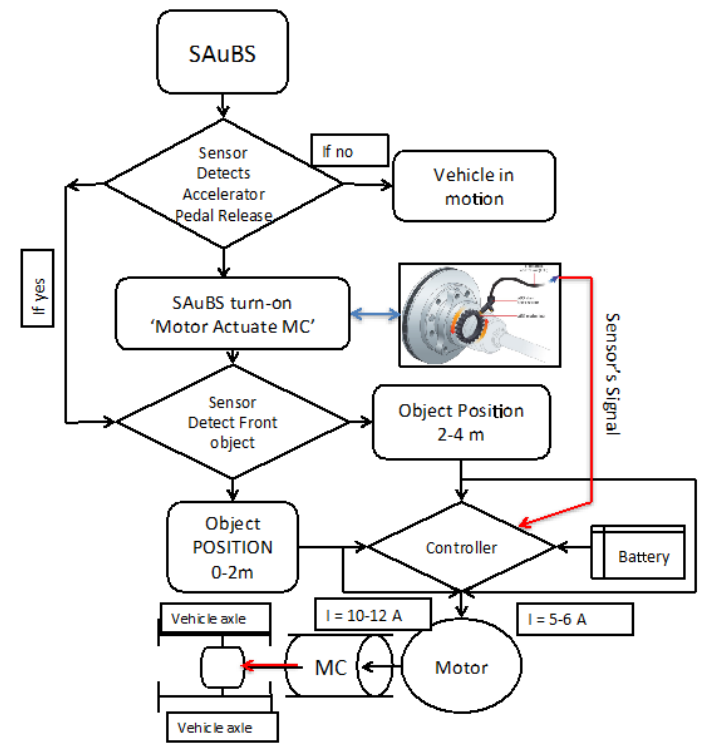

Figure 1: Flow of action of $S$ AuBS

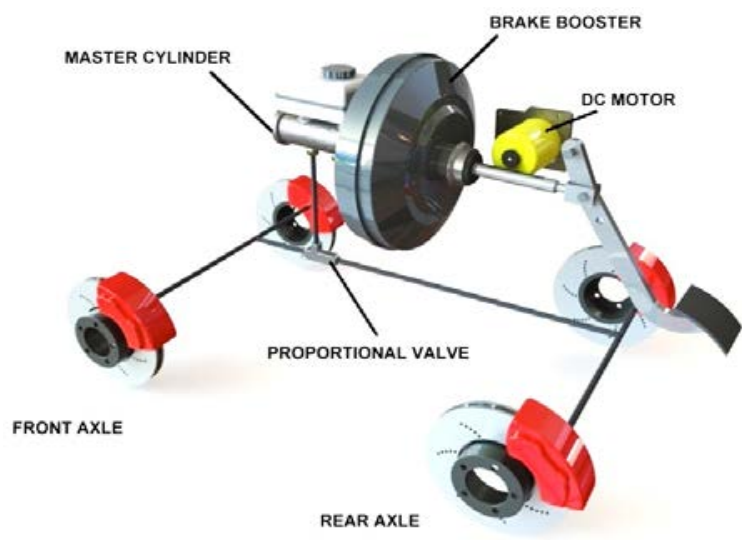

Figure 2: Architecture of Autonomous braking system

Performance characteristics of a road vehicle refer to its capability to decelerate and full-stop in a shorter distance. The braking torque required to decelerate or stop the vehicle can be estimated ${ }^{9,10}$ :

$T_{b(f)}=\frac{\mu_{b p} \times x_{f} \times P_{m c} \theta}{3}$

$T_{b(r)}=\frac{\mu_{b p} \times x_{r} \times P_{m c} \theta}{3}\left(r_{o}^{3}-r_{i}^{3}\right)$

where, $\mu_{b p}$ is the brake pad friction coefficient, $T_{b}$ braking torque of the rotor, $\mathrm{Nm}, P_{m c}$ master cylinder pressure, $\theta$ is the angular position of the brake pad from the centre of the rotor, $T_{t}$ traction torque of the vehicle, $\mathrm{Nm}, x_{r}$ is the percentage of $P_{m c}$ to the front axle and $x_{r}$ is the percentage of $P_{m c}$ to rear axle. The suffix $f$ and $r$ represent front and rear axle and $o$ and $i$ indicate the outer radius and inner radius of the brake pad from the centre of the braking rotor.

a. Rotor barking torque to decelerate the car,

$T_{b(f)}(t)+T_{b(r)}(t)<T_{x}$

b. Rotor braking torque to a full stop the car, 
$T_{b(f)}(t)+T_{b(r)}(t) \geq T_{x}$

where, $T_{x}$ is the traction torque, $\mathrm{Nm}$.

\subsection{Generic Form Algorithm for the AuBS}

The braking power of the braking system in this study has been assigned based on the signal of the wheel speed sensor, ultrasonic sensor and accelerator sensor. The vehicle starts to decelerate when the accelerator pedal releases and the master cylinder starts to develop the braking pressure for the braking devices (either disk or drum braking). The braking devices develop the actuation force which causes the development of braking force at the brake pad and the vehicle decelerates or full stop. Based on the condition of the braking force development, the genetic algorithm has been developed, $B_{f} \geq 0$. Braking force is subjected to instantaneous signal of:

i. Wheel speed sensor,

$\operatorname{wss}(t)=\dot{x}, 0 \leq \dot{x} \leq 120(\mathrm{~km} / \mathrm{h})$

ii. Ultrasonic sensor signal,

$u s(t)=x, 2 \leq x \leq 5(m)$

Rotor speed, $M_{r}(t)=\oint\left(I_{b}\right)$

Braking force, $B_{f}=\oint\left(\right.$ rpm $\left._{w s s}\right)$

Error, $e=M_{r}-M_{a}$

Rate of error, $\dot{e}=\frac{d}{d t}\left[M_{r}-M_{a}\right]$

where, $\dot{x}$ is the speed of the wheel, $\mathrm{km} / \mathrm{h}, x$ displacement based on the signal of ultrasonic sensor, $m, e$ error between rotor rpm and wheel actual rpm, $\dot{e}$ is the rate of error, $M_{r}$ rotor rpm, $M_{a}$ actual $r p m$.

Figure 3 shows the controlling action of the AuBS using ANFIS to decelerate the vehicle starts when the driver to lift-up foot from the acceleration pedal. The system then starts working with the input from the ultrasonic sensor and wheel speed sensor, send to the controller, which controls the power flow to the motor to actuate the MC in the development of hydraulic pressure to decelerate or fully stop the car without the driver any effort. When the car is at a higher or cruising speed, however, this system will not be useful to fully stop the vehicle.

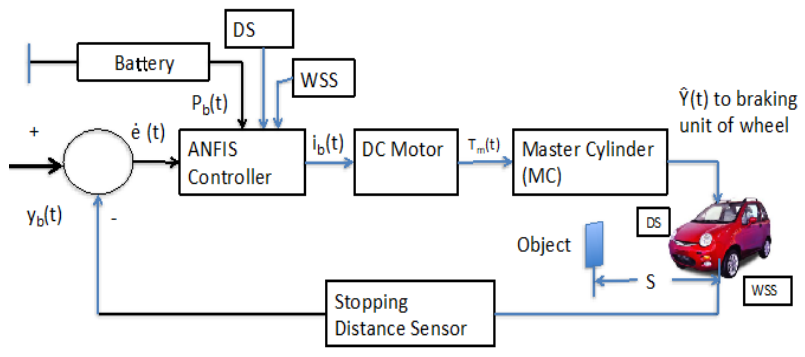

$P_{B}(t)$ - Instantaneous battery power to motor, $i_{b}(t)$ - battery current, $T_{m}(t)$-motor

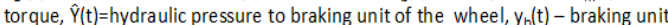
pressure, WSS - wheel speed sensor, DS-distance sensor, S-stopping distance.

Figure 3: Basic block diagram of a closed-loop vehicle braking system

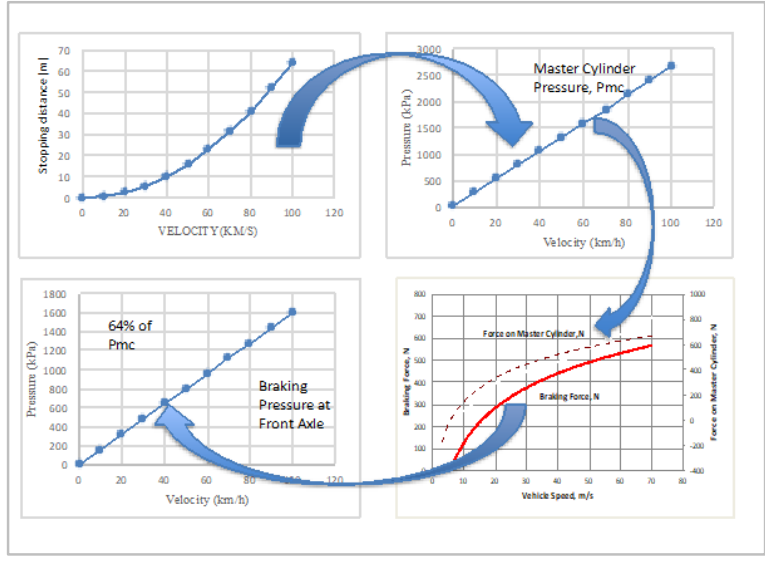

Figure 4: Fundamental of braking system

Figure 4 depicts the basic braking characteristics that must be met by the vehicle's braking system during deceleration or full stop. It is also critical to avoid a vehicle collision. The diagram depicts the vehicle's safe braking distance at various speeds, as well as the required force on the MC and hydraulic pressure on the axles. The related force on the MC will be roughly $510 \mathrm{~N}$ if the vehicle's safe stopping distance is $30 \mathrm{~m}$ at $40 \mathrm{~km} / \mathrm{h}$, allowing the hydraulic pressure to rise to $1100 \mathrm{kPa}$. As a result, the hydraulic pressure at the front axle will increase by around 64\%. A pressure of $620 \mathrm{kPa}$ is $(620 \mathrm{kPa})$.

\section{CONTROL STRATEGY OF SAUBS}

An adaptive neuro-fuzzy inference system (ANFIS) is a type of artificial neural network that uses the TakagiSugeno fuzzy inference system. It combines neural networks and fuzzy logic concepts, allowing it to capture the advantages of both in a single framework. Its inference system is made up of a set of fuzzy IF-THEN rules with the capacity to approximate nonlinear functions through learning. The best parameters obtained by a genetic algorithm can be used to make the ANFIS more efficient and ideal.

\subsection{ANFIS Structure}

Layer 1 The inputs to the nods in the first layer are the fuzzy sets of the ANFIS. Every node in this layer is an adaptive node. The layer is connected between the input terminal and its corresponding membership function nodes.

Layer 2: This layer's nodes are all circle nodes labelled $\Pi \square$ It multiplies the input signals and sends the final result out. For example,

$w_{i}=\mu a_{i}(x) \times \mu b_{i}(y), i=1,2 ;$

where, $\mu a_{i}$ is the membership function of linguistic variable $a$ and $\mu b_{i}$ is the membership function of variable $b, a_{i}$ is the linguistic level (very small, VL, small L, M medium, $\mathrm{H}$ high and $\mathrm{XH}$ extreme high) and similarly for $b_{i}$ (VL, L, M, H, and XH). Each node output represents the firing strength of a rule.

Level 3: $\mathrm{N}$ is the label for each node in this layer is assigned by $\mathrm{N}$. The i-th node determines the ratio of the firing strength of the i-th rule to the total firing strength of all rules: 
$\bar{w}_{i}=\frac{w_{i}}{w_{1}+w_{2}}, i=1,2$;

Here, $\bar{w}$ is the normalized fring strength of rule $i$.

Layer 4: This layer node is an adaptive node, connected to the corresponding normalized node in the previous layer, and receive inputs from $x$ and $y$. Each node in this layer is a local linear model of the Sugeno fuzzy system; integration of outputs of all local linear model yields predicted output:

$\bar{w}_{i} p_{i}=\bar{w}_{i}\left(r_{0}+m_{r} x+n_{i} y\right)$

Layer 5: The single node in the layer is a fixed mode levelled $\Sigma$, which computes the overall output as the summation of all incoming signals:

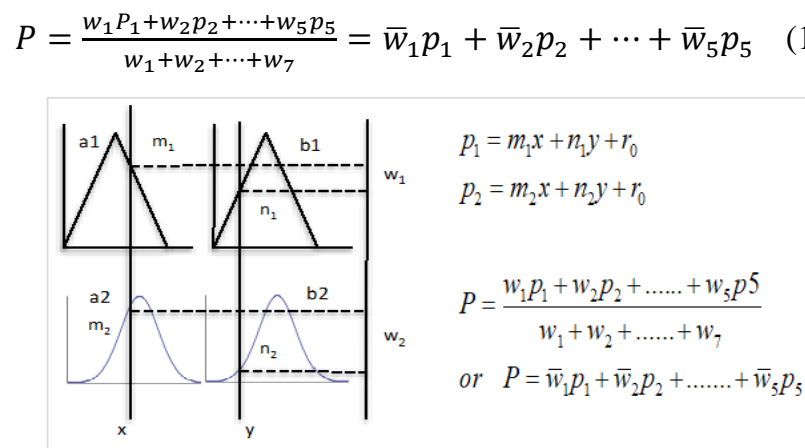

(a)

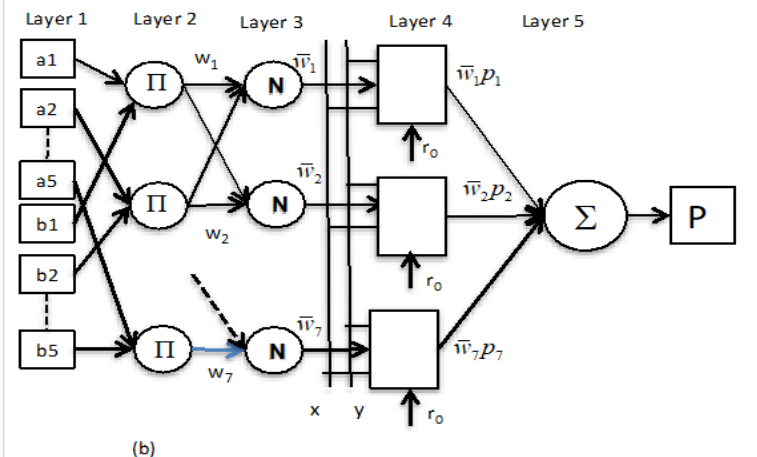

Figure 5: ANFIS model classification

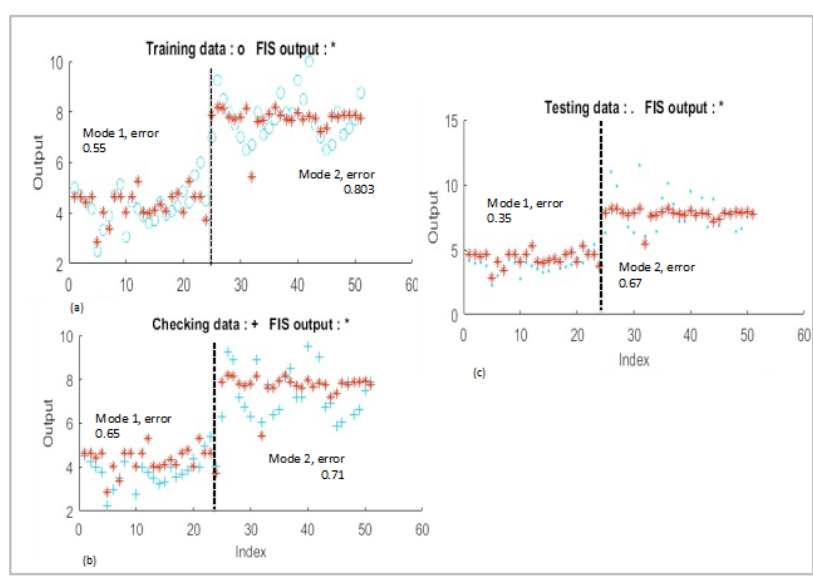

Figure 6: Theoretical ANFIS model performance

\subsection{Training, Checking and Testing of the ANFIS model}

The ANFIS model is trained, checked, and tested using the
MATLAB software program, which allows for easy manipulation of the model's parameters and variables. The system receives the entire set of data on the vehicle's motor speed, sinkage, and power requirement, is divided into three parts:

i. Training data, which made up $69.8 \%$ of the total 102 data set, enabled so-called "learning with a supervisor," in which the network's output is known ahead of time for proper inputs. The least error of training data as shown in Figure 6 (a) is 0.55 for mode 1 (deceleration), 0.803 for mode 2 (full stop), and 0.69 for overall.

ii. Checking data, which is primarily intended to prevent over-fitting of training data. In each training period, the ANFIS model monitors the value of the checking error. As illustrated in Figure 6 (b), the overall error is 0.83 . The checking data makes up $50 \%$ of the total data set. The checking data has been made using MATLAB Simulink.

iii. We used testing data to evaluate the ANFIS model. The output of the ANFIS model is compared to known values in order to discover the least amount of inaccuracy possible. The smallest inaccuracy was 0.23 , as shown in Figure 6(c). Testing data accounted for nearly $50 \%$ of the total data set. Data for testing was gathered from a field experiment.

Figure 4 also shows that the fuzzy interference data is more correlated compared to the training data and checking data. This is because of the ANFIS interpretation capability and the ease of encoding a priori knowledge.

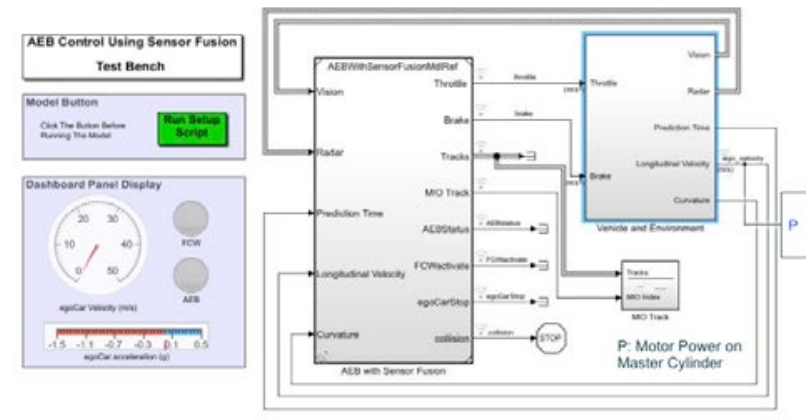

Figure 7: ANFIS trained AEM model

A MATLAB ANFIS controller Simulink model has been designed to optimize the braking pressure of the master cylinder to deceleration/full stop of the vehicle. The working range for the controller has shown in Table 1 which has been identified from the ANFIS MATLAB model.

\section{CASE STUDY: VEHICLE IN PEDESTRIAN}

\subsection{AEB Model Study- Pedestrian Crossing}

The Autonomous Emergency Braking (AEB) technology assists drivers by providing sufficient braking force to avoid a crash. This system uses an obstacle positioning sensor and a speed sensor, and the system's output is braking force, which can come from the action motor power that applies force on the master cylinder which produces the braking pressure. The Adaptive Neuro-Fuzzy 
Inference System (ANFIS) is a technology that may be used to train a controller that mimics human behavior using current experimental data. When a data set is entered into ANFIS, membership functions are generated, which are then trained into a set of rules that can be satisfied. ANFIS will train an AEB controller in this project, resulting in a system that can autonomously mitigate any road collision. Figure 7 shows the ANFIS Matlab model to simulate the optimum braking system effective parameters with trained AEM model data.

Table 1

Working condition for AuBS

\begin{tabular}{lccc}
\hline \hline $\begin{array}{l}\text { Vehicle } \\
\text { Dynamic } \\
\text { mode }\end{array}$ & \multicolumn{2}{c}{$\begin{array}{c}\text { Distance between } \\
\text { vehicle }(\boldsymbol{m})\end{array}$} & $\begin{array}{c}\text { Force } \\
\text { applied at } \\
\text { MC }(\boldsymbol{k N})\end{array}$ \\
\cline { 2 - 3 } & Front & Side & \\
\hline Power brake & $2.5-2.0$ & 0.0 & $0.9-1.7$ \\
Deceleration & $3.5-2.5$ & $0.0-0.5$ & $0.0-0.9$ \\
Cruising & $4.0-3.5$ & $0.51-$ above & 0.0 \\
\hline \hline
\end{tabular}

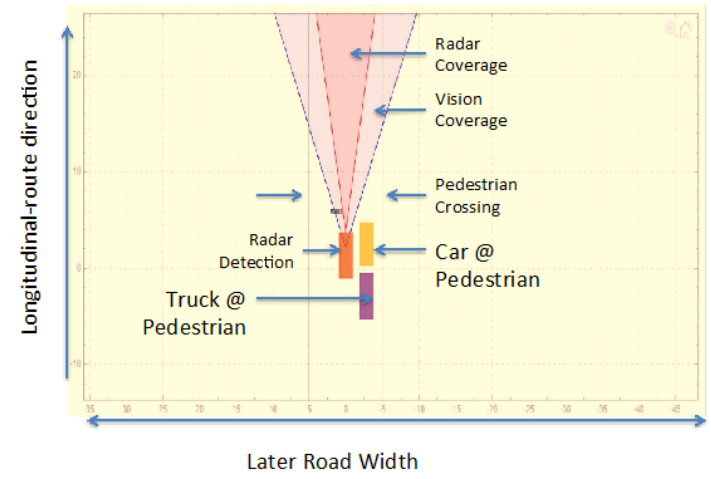

Figure 8: Pedestrian crossing

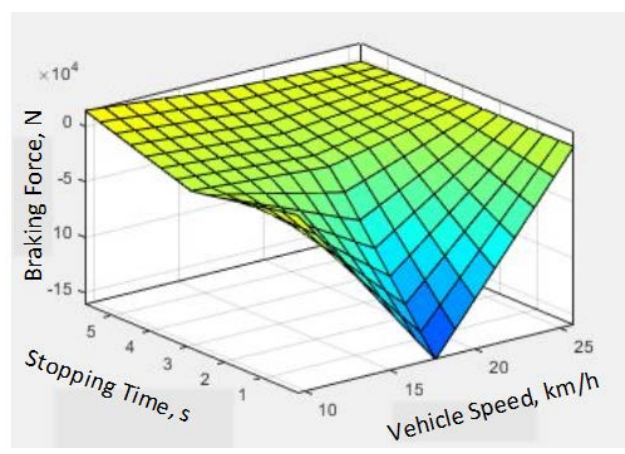

Figure 9: ANFIS simulation result for pedestrian

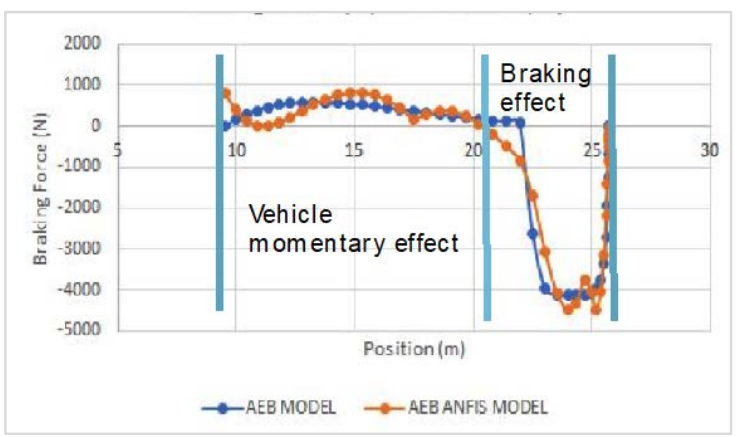

Figure 10: Comparison of ANFIS model with AEB model

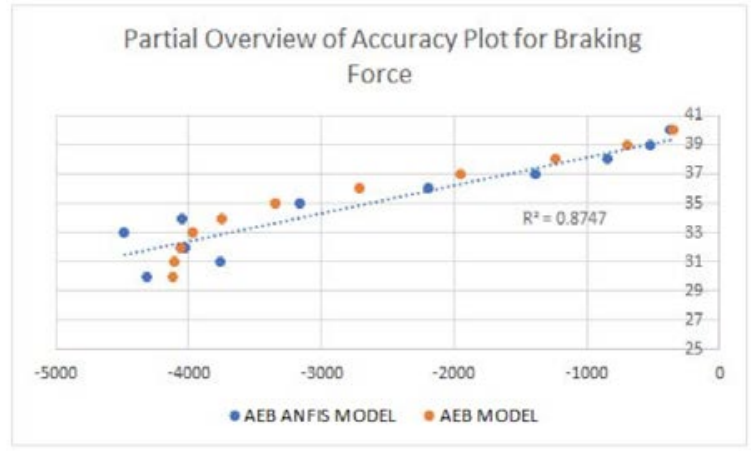

Figure 11: ANFIS result is verified with the AEB model result

The pedestrian crossing and the location of the car recognized by the RADAR SENSOR are visualized in Figure 8. Figure 9 shows the ANFIS model is being simulated with radar sensor input and compared with the AEM model in Figure 10. In terms of generating braking force, Figure 9 shows how the ANFIS model outperforms the AEB model. The ANFIS controller will activate the braking system just before the crossing, causing the automobile to slow down or stop completely. Figure 11 depicts the 88 percent discrepancy between the AEM and ANFIS results.

\section{AEB MODEL TESTING - EXPERIMENTALLY}

A laboratory $1 / 4$ scale prototype of AuBS as shown in Figure 12, was constructed using a typical scouter braking system, which includes the master cylinder, brake pad, brake callipers, and brake tube (Figure 12). The performance of the prototype has been conducted using a $155 \mathrm{~kg}$ load, road adhesion, $\mu_{\mathrm{p}}=0.6$, frictional coefficient of brake pad, $\mu b p=0.46$, and master cylinder bore of $2.85 \mathrm{~cm}$.

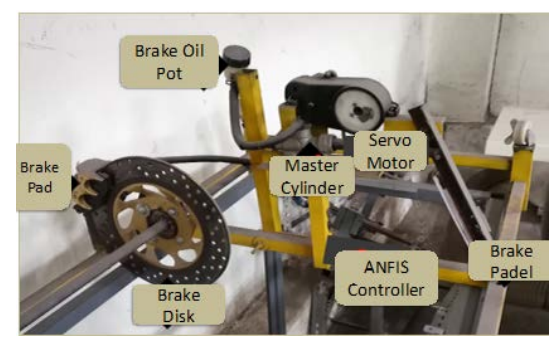

Figure 12: A laboratory scale AuBS
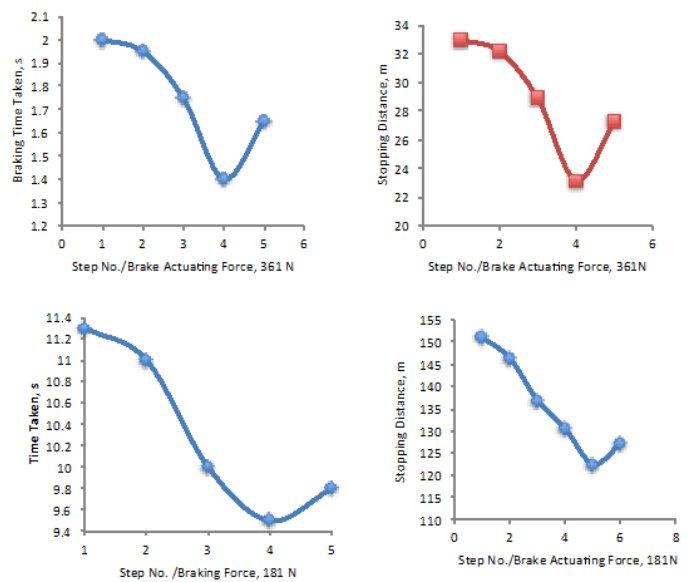

Figure 13: ANFIS controlled laboratory scale AuBS 
The experimental result of the laboratory scale AuBS controller equipped brake system is shown in Figure 12. The servo-motor applied forces of $181 \mathrm{~N}$ and $361 \mathrm{~N}$ on the $\mathrm{MC}$ were used in the testing. The vehicle's stopping distance was found to be $23 \mathrm{~m}$ at time $1.4 \mathrm{~s}$ for the applied force $361 N$, while its braking distance was found to be $122 \mathrm{~m}$ at time $9.5 \mathrm{~s}$ for the braking force of $181 \mathrm{~N}$.

\section{CONCLUSIONS}

- Semi-Autonomous Braking System (AuBS) can be used as a stepping stone for the vehicle's braking system during traffic congestion to reduce the driver's input.

- ANFIS controlled braking system is more efficient than a conventional braking system at decelerating or fully stopping the vehicle without the need of the driver's support.

- Servo motor speed is inefficient to quickly control the vehicle, especially in GO/STOP mode dynamics and keep the vehicle in safe mode.

- $\quad$ AuBS adds value to the existing conventional braking system to control the vehicle based on the response of ultrasonic and photosensors in pedestrian crossings and slow-moving traffic.

- $\quad$ To justify the AuBS performance, more research is needed in the lab and the field with a full-scale AuBS model.

\section{ACKNOWLEDGEMENTS}

Authors would like to express their gratitude to the Dept. of Mechanical Engineering, IIUM, Kuala Lumpur, Malaysia. They also would like to thank the anonymous reviewers and editorial board members of MIJST for insightful comments and suggestions.

\section{REFERENCES}

Anbalagan P \& Suyambukanni N, “Non-Motorized Transport (with special reference to Bicycles in Chennai City)" Indian Journal of Transport Management, Oct-Dec 2010, Vol. 34(4), pp 273-284.

ASEAN NCAP, “Asean ncap new rating protocols for 2017-2020 - the new car assessment program for southeast asia," http://aseancap.org, accessed: 2017.08.26.

Berger, D. Block, S. Heeren, C. Hons, S. Kuhnel, A. Leschke, D. Plotnikov, and B. Rumpe, "Simulations on consumer tests: A systematic evaluation approach in an industrial case study,” IEEE Intelligent Transportation Systems Magazine, vol. 7, no. 4, pp. 24-36, 2015.
Breuer, A. Faulhaber, P. Frank, and S. Gleissner, "Realworldsafety benefits of brake assistance systems," in 20th International Technical Conference on the Enhanced Safety of Vehicles (ESV), no. 07-0103, 2007.

Distner, M. Bengtsson, T. Broberg, and L. Jakobsson, "City safetya system addressing rear-end collisions at low speeds," in Proc. 21st International Technical Conference on the Enhanced Safety of Vehicles, no. 09-0371, 2009.

Fildes, M. Keall, N. Bos, A. Lie, Y. Page, C. Pastor, L. Pennisi, M. Rizzi, P. Thomas, and C. Tingvall, "Effectiveness of low speed au- tonomous emergency braking in real-world rearend crashes,” Accident Analysis \& Prevention, vol. 81, pp. 24-29, 2015.

Fridulv Sagberg (1999), „Road accidents caused by drivers falling asleep"e, Accid. Anal. Prev., Vol. 31, pp.639-649.

Hassen, A., Godesso, A., Abebe, L., and Girma, E. (2011). Risky driving behaviours for road traffic accident among drivers in Mekele city, Northern Ethiopia. Research Notes, 4, 535.

Hamid, K. Pushkin, H. Zamzuri, D. Gueraiche, and M. A. A. Rahman, "Current collision mitigation technologies for advanced driver assistance systems-a survey,” PERINTIS eJournal, vol. 6, no. 2, 2016.

Hamid, M. H. M. Ariff, H. Zamzuri, Y. Saito, M. A. Zakaria, M. A. A. Rahman, and P. Raksincharoensak, "Piecewise trajectory replanner for highway collision avoidance systems with safe-distance based threat assessment strategy and nonlinear model predictive control,” Journal of Intelligent \& Robotic Systems, pp. 1-23, 2017.

Mittal, N. SIMBA Project, "Policies and Programs for road safety in developing India”, Division of Environment and Road Traffic safety, Central Road Research Institute, New Delhi, India, 2008.

Paine, D. Paine, C. Newland, and S. Worden, "Encouraging safer vehicles through enhancements to the ncap rating system," in 22nd International Technical Conference on the Enhanced Safety of Vehicles (ESV), no. 11-0107, 2011.

Rahman, A. and Mohamad Amsyar (2018) DC motor amplified mechanical-hydraulic braking system for passenger car. Journal of Applied Science, Vol.18(2): pp.56-64.

Rahman, Mohiuddin AKM and Ahsan Sakif (2018) Development of electro-hydro automatic parking braking system: Automotive use. International Journal of Engineering and Technology., Vol.7(6S), 264-269.

Rahman, A. and Mohamad Amsyar (2018) DC motor amplified mechanical-hydraulic braking system for passenger car. Journal of Applied Science, Vol.18(2): pp.56-64.

Rahman, A, Nurul Hasan, Mohd Zaki (2020) Modelling and Validation of Electric Vehicle Drive Line Architectureusing Bond Graph. TEST Engineering and Management, Vol.82 (Jan-Feb, 2020), pp.15154-1516.

Zwahlen, C. Jackowski, and M. Pfa"ffli, "Sleepiness, driving, and motor vehicle accidents: A questionnaire-based survey," Journal of forensic and legal medicine, vol. 44, pp. 183-187, 2016. 